\title{
Development of Land Resource Management Data System Based on MAPGIS
}

\author{
Hao Tao ${ }^{1, \text { a }}$ \\ ${ }^{1}$ School of Land Sciences and Technology, China University of Geosciences(Beijing), Beijing, China \\ ataohaozgdz@163.com
}

Keywords: land resource management; GIS; MAPGIS

\begin{abstract}
MapGIS is an implementation of remote sensing processing and GIS fully integrated to support air, ground, and surface, underground true three-dimensional space integration GIS development platform. The task of the management of land resources data system is grasp timely, fully and correctly the utilization status of the land resources of great significance for strengthening the management of land resources. The paper presents the development of land resource management data system based on MAPGIS. The MAPGIS software platform can from remote sensing images for land resources information to help the digital land resource management, modern and scientific.
\end{abstract}

\section{Introduction}

Land resource management refers to the countries under certain environmental conditions, the integrated use of administrative, economic, legal, technical methods, to improve land use ecological, economic, social, and maintenance of the dominant land ownership in society, to adjust the relationship to the land, supervision of land use, of planning, organization, coordination and control of integrated activities.

With the continuous improvement of remote sensing data spatial resolution, the amount of data is increasing, large-scale, high-speed real-time interpreter work more and more dependent on computer processing. Interactive interpretation of interpretation methods makes computer complement each other, give full play to their strengths can be accurately and effectively interpret the remote sensing images. On this basis, choose the appropriate GIS software allows us to more quickly process and application of these remote sensing data; make it converted to the urgent need to be applied information.

The use of remote sensing images as a data source to extract land use / land cover information, commonly used in the MAP FO N ARCV IEW, in ARC / the I FO and other foreign professional soft $\mathrm{N}$, less use of domestic professional software [1]. MAPGIS software is outstanding in the domestic GIS software with independent intellectual property, software design and development are linked closely with the national standards recommended by the Ministry of Land and appraisal certification and platform, has been widely used in China's urban planning, surveying and mapping, land, real estate, tourism and other fields, has now become our software of choice for the field of digital construction.

Management of land resources database system towel data including vector data, image data and text report data, a huge amount of data, different levels of rapid retrieval mechanism is automatically determined in accordance with the level of browsing technology to display the corresponding content to the image data of the sea wall fast seamless browsing. Statistics and analysis of data query to update the data query system can support a variety of ways to search. Including point query Administrative Region of the query, buffer Hong inquiry polygon query a variety of spatial query: You can also attribute field to query, fuzzy query, the combination of query multi-granularity cross-storage unit data query and polygons historical changes in retrospective inquiries; and is able to save Lian consultation results and output. 
According to the geographic features hierarchical management requirements, the interpretation can in the "Layers" menu in advance edit a good layer names dictionary, then use the drawing tools of point, line, and the district in the different thematic graphics rendering of an independent view topic, so that a thematic layer. Legend plate is an important tool used to standardize the vector data MAPGIS platform, a quick tool for graphical editing process. Applications legend plate mapping can be done pixel parameter is automatically specification to meet the requirements of hierarchical vector of, but also to avoid the duplication of the parameters of the drawing elements of the vector process. In addition, the topology error checking MAPGIS software can also batch suspension node and quickly set up the topological relationship between the polygons, Application of MAPGIS software powerful and convenient operation of functions greatly facilitate the interpretation of remote sensing images of the human-computer interaction to interpret the work, improve work efficiency. The paper presents the development of land resource management data system based on MAPGIS.

\section{MapGIS system model}

MapGIS in the Digital Group, product names, geographic information system with completely independent intellectual property rights in China is the world's only integrated development of structures GIS data center platform to realize remote sensing processing and GIS fully integrated to support air, ground, surface underground the whole space is really three-dimensional integration of GIS development platform.

The system uses a service-oriented design ideas, multi-tier architecture, data organization for spatial entities and their relationships, efficient massive data storage and indexing of large-scale multi-dimensional dynamic spatial information database, 3D solid modeling and analysis spatial data processing capability, can support the distributed computing of spatial data in the local area and wide area network environment to support distributed spatial information distribution and sharing of spatial information services of the network can support large, distributed national space infrastructure facilities.

MapGIS framework include: the development platform, tools, products and solutions. Development platform, including server development platform (DC Server), Remote Sensing Processing Development Platform (RSP), the 3D GIS development platform (TDE), Internet GIS services development platform (IG Server), embedded development platform (EMS), data center integrated development platform and wisdom of industry integrated development platform for partners specializing in the field of application development [2].

Tool products covering a wide range of industries, including vector data processing tools, and remote sensing data processing tools, land and tools products, municipal and tools products, 3D GIS tools products, it is real estate tools and embedded tools. The solution is the development platform, requirements documents, design documents, and document an integrated service. MapGIS in 3D GIS / remote sensing, digital city / digital municipal, land / agriculture, forestry, communications / broadcasting / postal areas have to use the same time of WebGIS, "Jindun two" of PGIS, forest fire prevention, real estate information management, quality supervision, etc. the industry also has the appropriate application solutions, as is shown by equation 1.

$$
W_{j}^{\lambda} f(x, y)=f \times \Psi_{j}^{\lambda}(x, y)=\iint_{R^{2}} f(u, v) \Psi_{j}^{\lambda}(x-u, y-v) d u d v
$$

MapGIS K9 SP3, "cloud services" super engine take suspension architecture, scalability, strong self-adaptability; function data is the separation between the features and functionality, functionality and data between the loose coupling; very strong vitality, a cloud cells out of the problem, new cloud cells can be gathered at any time come to replace; a cross-platform, high scalability characteristics, can be easily shared data between different devices or applications, you can facilitate the organic integration with ERP, CRM, Business Intelligence (BI) and other enterprise systems to provide users with timely, efficient, and customized GIS services. 
Version management and conflict is detection mechanism version of the long transaction mechanism. Management and control of network topology data model-based workflow engine, flexible adjustment and customization of business to address the seamless integration of GIS and OA [3]. Standard adaptive spatial metadata management system, metadata collection, storage, building a database, query and share release, it is support the SRW protocol, with the distribution between the cable capacity.

The system has the following characteristics: a distributed cross-platform, multi-storey multi-level architecture, design ideas for the "service", with the spatial data model for geographical entities, describe the spatial characteristics of arbitrary complexity and non-spatial characteristics full space to express, non-space, physical space, symbiotic, the multiplicity of relations. With massive data storage and management capabilities, vector, raster images, three-dimensional four-in-one mass data storage, and efficient spatial index., as is shown by figure 1.

\begin{tabular}{|l|r|r|}
\hline-0.1 & -0.2 & -0.3 \\
\hline-0.1 & -0.2 & -0.3 \\
\hline-0.1 & -0.2 & -0.3 \\
\hline
\end{tabular}

\begin{tabular}{|l|l|l|}
\hline 0.1 & 0.2 & 0.3 \\
\hline 0.1 & 0.2 & 0.3 \\
\hline 0.1 & 0.2 & 0.3 \\
\hline \multicolumn{3}{|l}{} \\
\end{tabular}

Figure. 1 MapGIS K9 SP3 example

Support for true three-dimensional modeling and visualization, three-dimensional mass data storage and management, to quickly build 3D professional model, three-dimensional data visualization and fusion analysis [4]. Provide spatial information application services based on SOAP and XML, follow Open is specification, support for WMS, WFS, WCS, GLM3.Support Internet and wireless Internet, and supports a variety of smart mobile devices.

Graphical input operation is relatively simple, reliable, able to adapt to engineering requirements MAPGI S digitizer input and scan input to a variety of input means, to automatically track segment, node adjustment, cropping and extension of the line junction point and polygon topology automatically generated drawing deformation non-linear correction, and automatic detection of the error, thus greatly simplifying the graphical input operation, to ensure the reliability of the input, in particular, apply to the input of relatively large engineering drawings, as is shown by equation 2 .

$$
\begin{cases}D_{j, F}^{\xi}(m, n)=D_{j, A}^{\xi}(m, n) & E_{j, A}^{\xi}(m, n) \geq E_{j, B}^{\xi}(m, n) \\ D_{j, F}^{\xi}(m, n)=D_{j, B}^{\xi}(m, n) & E_{j, A}^{\xi}(m, n)<E_{j, B}^{\xi}(m, n)\end{cases}
$$

MAP GIS use is widespread; according to of MAPGIS the functional and technical features, it can play a larger role in the following five aspects. A. The outstanding advantage of the multi-source data collection and integration of the MAP GIS can easily receive and collection of different media, different types and formats of data. Field measurement records, hand made sketches, formal base map, aerial photographs, remote sensing, digital image, and professional data, or GPS real-time positioning data, it can receive and acquisition. Whether they are in the form of graphics, images, text, digital, or video, regardless of their data formats are the same, the MAP of GIS can manage them with a unified database, so as to facilitate the comprehensive analysis of the source data.

Windows as a platform, using $\mathrm{C}++$ language development, user-friendly, easy to use. Scanner input and digitizer input means, a complete error, and the error correction method. Have a rich graphical editing tools and powerful graphics capabilities [5]. Intuitive and practical attributes dynamically defined editing features and multimedia data, the plug-in database management capabilities. To library management system has a strong map mosaic, management, roaming and flexible cross-maps search capabilities, and you can manage up to thousands of maps. 
MAP GIS has strong spatial analysis and query capabilities. Therefore to learn workers can easily use the interactive mode of sources of information were compared, analysis, and gain new inspiration and knowledge to improve and summarize the law in order to facilitate planning, decision-making and operations. The Geosciences process simulation, geographic information system analysis and forecasting can not only static spatial relations of spatial entities, but also reflect changes in the spatial entity with the time and space, in our study tectonic movements, land use, soil erosion, urbanization development and other issues, we can be two or more different times of the status quo diagram space superposition analysis or dynamic display, so we can effectively carry out the process of simulation, analysis and forecasting.is defined as equation 3.

$$
\left\{\begin{array}{l}
D_{j, F}^{\xi}(m, n)=-\left(\left|w_{j, \text { max }}^{\xi}(m, n) D_{j, A}^{\xi}(m, n)\right|\right. \\
\left.+\left|w_{j, \text { min }}^{\xi}(m, n) D_{j, B}^{\xi}(m, n)\right|\right) \quad D_{j, A}^{\xi}(m, n)<0 \\
D_{j, F}^{\xi}(m, n)=\left|w_{j, \text { max }}^{\xi}(m, n) D_{j, A}^{\xi}(m, n)\right| \\
+\left|w_{j, \text { min }}^{\xi}(m, n) D_{j, B}^{\xi}(m, n)\right| \quad D_{j, A}^{\xi}(m, n) \geq 0
\end{array}\right.
$$

MAPGIS6.5 many new features: 1. the full support of the Orca database. Two field measurement systems is more powerful handheld mapping system via the Pocket PC is quick and easy field electronic tablet mapping [6]. (3) On the scanned topographic maps of various scales by the grid precision correction to generate the precision of standard DRG data. DRG quality checks provide a sound; can be interchangeable with a variety of image data formats. 4 secondary development libraries to further improve and provide a more complete secondary development libraries, AP I function more complete, the class library to develop more flexible and comprehensive support MAPGIS components and controls development, as is shown by equation4.

$$
\begin{aligned}
\tilde{V}_{j-1} & =V_{j-1} \otimes V_{j-1}=\left(V_{j} \oplus W_{j}\right) \otimes\left(V_{j} \oplus W_{j}\right) \\
& =\left(V_{j} \otimes V_{j}\right) \oplus\left[\left(V_{j} \otimes W_{j}\right) \oplus\left(W_{j} \otimes V_{j}\right) \oplus\left(W_{j} \otimes V_{j}\right)\right]
\end{aligned}
$$

Configure and manage the MAPGIS with the ORACLE database management procedures began to create a management process that automatically search the MAPGIS library already established in the corresponding database, if you do not exist to create stored procedures, management information table, the lock management table and other database objects. When the green hook into the blue hook, indicating that the established the MAPGIS management process is complete, after you can directly to use MAPGISDBA account login for spatial data management, without having to re-establish the management process. Note: (1) to configure the MAPGIS network management process needs to be installed SQLPLUS components (when installing the client must select this option).

\section{Development of land resource management data system based on the MAPGIS}

Selected based on can be seen the MAPGIS land resources, remote sensing interpretation of human-computer interaction from the above workflow, MAPGIS software from remote sensing images, it supports JPGP a variety of graphics, TIFF, BM TXT Image File Format, the sharing of resources. And E00G, exchange of various data formats, enabling data DW of SHP function, can achieve the precise positioning of the image, which is essential in the preparation of the sources of information and interpretation of polygons painting on stage. Reduced functionality of the software is the overall situation, thereby increasing the overall analysis of remote sensing images, and help operators to make more accurate interpretation.

MAPGIS original application, mining engineering geology in engineering geology exploration data can be based on MAPGIS spatial database and efficient storage management. MAPGIS can be effective management of mining engineering geological maps and graphics and its properties associated with the key problem is that the graphical representation of the stronger of the editing 
capabilities. The MAPGIS can like CAD to draw the histogram in the mining resource development; can also use the drilling data and the histogram, or space-based database, MAPGIS profile and contour map can be drawn automatically. Slope control and dewatering of mine drainage, the MAPGIS geographic information systems technology can help mine workers to solve the mine dewatering and drainage the stop Slope design and stability analysis of engineering problems.

These advantages are the traditional visual interpretation and computer-based remote-sensing images automatically analyze the treatment does not have it. Practice has proved that MAPGIS software platform, using the method of access to land resources information from remote sensing image interpretation of human-computer interaction, enhance the image interpretation accuracy and efficiency, help achieve the digitization of land resources management, modernization and scientific.

WEB GIS technology is in order to establish data sharing. Avoid waste of resources. 4 Conclusion With the regional economic and social Fat Yiu and urbanization continue to expand, land resources is the high cost of land, efficient, reasonable, and intensive use of every inch of land, and efficient system juice. Analysis and scientific planning, it is decision-making use of every piece of land. Real-time monitoring of land use status quo, to keep 18 million mu of arable land red line, a series of problems has emerged. However, it is geographic information systems (GIS), remote sensing (Rs) and Global Positioning System (GPS) "3S" integrated technology continues to evolve, has become a generic technology for land resource management, as is shown by equation5.

$$
S N R=10 \times \log _{10} \frac{\sum_{i=1}^{M} \sum_{j=1}^{N} F\left(x_{i}, y_{j}\right)^{2}}{\sum_{i=1}^{M} \sum_{j=1}^{N}\left[R\left(x_{i}, y_{j}\right)-F\left(x_{i}, y_{j}\right)\right]^{2}}
$$

Within a certain depth and height of the land, attached too many of the breeding of all production capacity, such as containing a variety of soil nutrients and moisture. Bite the air, but also to accept the sunlight, light, heat, etc., these are on Earth, biological growth, the basic conditions for breeding. Without these environmental conditions and their functions. Life on Earth can not be growth and development; human power can not survive and develop. Human resources (intangible) function to be of material production, in addition to the need for biological resources, you also need the non-material resources of a large Land. Windows XP operating system using Visual C \#.net to achieve the above rule set and computing algorithm. For randomly generated data sets, the relationship between the probability of $75 \%$, we do the extended test, the number of objects in increments of 12 , recorded by the sub-formal context. The paper presents the development of land resource management data system based on the MAPGIS, the results shown in Figure 2.

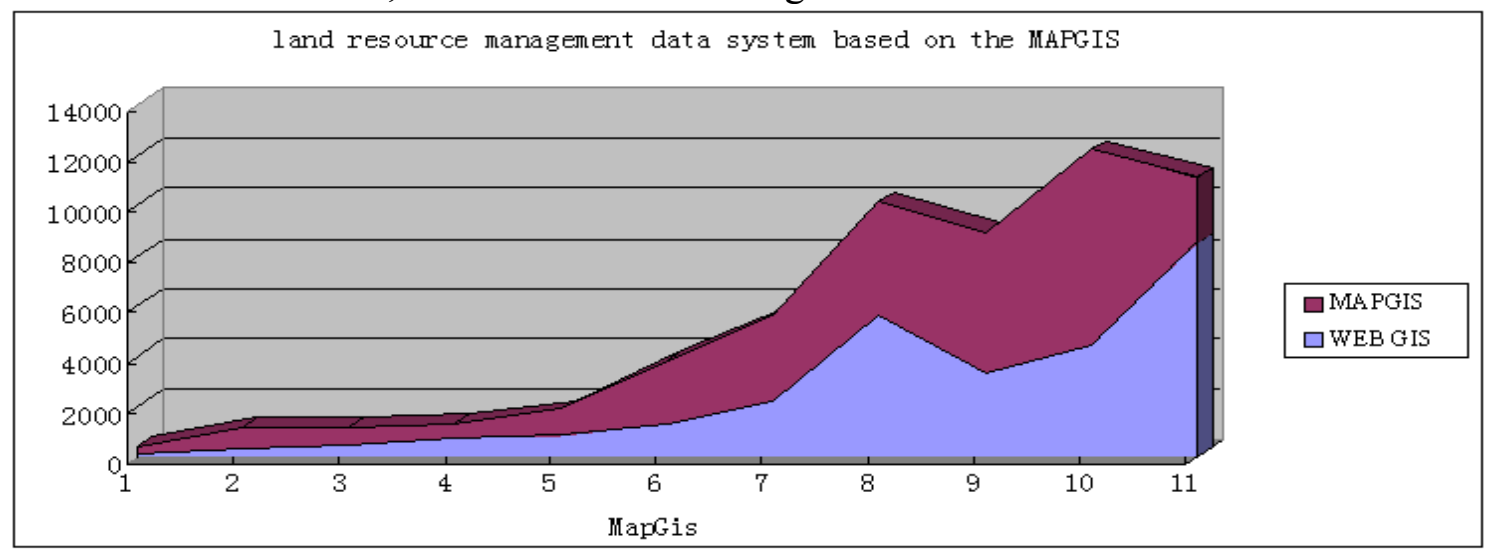

Figure. 2 The land resource management data system based on MAPGIS compared to WEB GIS map

Provides an important technical foundation is in order to achieve sustainable land development. Its function and role is mainly reflected in the following areas: (1) to provide spatial analysis models and results of the analysis technology in the study area land sustainable development strategy must be 
social, economic and environmental as a whole. The economic system has always been considered non-space, the need to introduce the new concept of spatial analysis and environmental analysis. "3S" technology for economic development, regional studies, space impact analysis, thereby enhancing regional economic, it is social and environmental integrity. (2)Temporal analysis and development of sustainable land use is in order to the basic elements of predictability. At present, all large construction projects with the geological survey determination of the boundary $\mathrm{T}$ for the use of MAPGPS, to ensure the smooth implementation of the construction project.

\section{Summary}

The paper presents the development of land resource management data system based on the MAPGIS. Existing cartographic software, MAPGIS software can input, editing, topology integration of spatial data digitized, with the powerful mapping capabilities can be achieved post-disaster reconstruction of land use planning to digitize drawings to establish a combination of graphical and attribute data database, map data layer management; flexible query, edit, statistics and analysis of the map information. With the MAPGIS the software, can greatly shorten the reconstruction of land use planning mapping preparation cycle; improve the value of the plan.

\section{References}

[1] Yingxia Wu, Jiejun Huang, Yanbin Yuan, Wei Cui, Yunjun Zhan, "Simulation of Land Use/Cover Change for Wuhan City Based on GIS and CA", JCIT, Vol. 7, No. 1, pp. $253 \sim 260$, 2012

[2] Xu Bing, Zhang Ying, Li Jing, "Dynamic Monitoring System of Sea Area Utilization based on 3S Technology", JCIT, Vol. 7, No. 9, pp. $413 \sim 421,2012$

[3] Guijuan Jiang, Xiaoxian Zheng, Yang Hu, "Forest health assessment in the Badaling Forest Farm of Beijing based on GIS and RS", JDCTA, Vol. 6, No. 10, pp. 87 93, 2012.

[4] Jinqu Zhang, YunQiang Zhu, Juanle Wang, Jiulin Sun, Yuyue Xu, "Flash based WebGIS System and its Application in Monitoring and Evaluating China's Regional Development", JDCTA, Vol. 5, No. 5, pp. $285 \sim 295,2011$.

[5] Yuankai Huo, Yudong Zhang, Shuihua Wang, Lenan Wu, "Diffusion Tensor Image Registration Based on Demon-Affine Method and FSGA-BFGS Algorithm", JCIT, Vol. 6, No. 1, pp. 108 115,2011

[6] Haidong Zhong, Jie Yin, Jianping Wu, Shenjun Yao, Zhanhong Wang, Zhenhua Lv, Bailang Yu, "Spatial Analysis for Crime Pattern of Metropolis in Transition Using Police Records and GIS: a Case Study of Shanghai, China ", JDCTA, Vol. 5, No. 2, pp. $93 \sim 105,2011$ 